\title{
Viscoelastic multiscaling in immersed networks
}

\author{
J. L. B. de Araújo ๑, J. S. de Sousa, W. P. Ferreira ๑, and C. L. N. Oliveira $\oplus^{*}$ \\ Departamento de Física, Universidade Federal do Ceará, 60451-970 Fortaleza, Ceará, Brazil
}

(Received 4 April 2020; accepted 24 July 2020; published 7 August 2020)

\begin{abstract}
Rheological responses are the most relevant features to describe soft matter. So far, such constitutive relations are still not well understood in terms of small scale properties, although this knowledge would help the design of synthetic and biomaterials. Here, we investigate, computational and analytically, how mesoscopic-scale interactions influence the macroscopic behavior of viscoelastic materials. We design a coarse-grained approach where the local elastic and viscous contributions can be controlled. Applying molecular dynamics simulations, we mimic real indentation assays. When elastic forces are dominant, our model reproduces the Hertzian behavior of contact. However, when friction increases, it restores the standard linear solid model. We show how the response parameters depend on the microscopic elastic and viscous contributions. Besides, we show that the contact Hertz model is equivalent to the virial stress, an atomistic approach of the continuum Cauchy stress tensor. In addition, relaxation experiments were performed on polyacrylamide gels with an atomic force microscopic in order to validate our numerical relaxation curves. The bisacrylamide concentration in the gel has a clear impact on the equivalent elastic and viscous contributions of the sample. Moreover, our findings also suggest that the relaxation times, obtained in relaxation and oscillatory experiments, obey a universal behavior in viscoelastic materials.
\end{abstract}

DOI: 10.1103/PhysRevResearch.2.033222

\section{INTRODUCTION}

One big challenge in science and engineering is the socalled multiscale modeling, namely, how constitutive relations of a material depend on its smaller scale interaction and composition $[1,2]$. Such emergence problem, as described by P. W. Anderson [3], is even more remarkable in soft matter, where mesoscopic structures combine the atomistic and macroscopic frameworks and are responsible to the material elasticity and viscosity [4,5]. In fact, the link among features of different scales forming the matter is highly nontrivial and subjected to intense research [6]. The seminal work of H. Hertz about mechanical contacts, for instance, is still used nowadays to measure elastic properties of materials by analyzing how samples are deformed under applied stresses [7]. Although it is one of the most used models to investigate the stiffness of a material, it fails to relate the macroscopic behavior with its inner parts. Besides, well-known analytical approaches such as the Maxwell, Kelvin-Voigt, and standard linear solid models apply circuit analogies to propose a simple manner of how elastic and viscous terms mix up in viscoelastic materials [8]. Again, these rheological models neglect any downscaling analysis.

\footnotetext{
*lucas@ fisica.ufc.br

Published by the American Physical Society under the terms of the Creative Commons Attribution 4.0 International license. Further distribution of this work must maintain attribution to the author(s) and the published article's title, journal citation, and DOI.
}

Currently, many sophisticated methods have been developed to study the viscoelasticity of materials. Nanoindentation experiments, for instance, such as those with atomic force microscopy, are extensively applied to investigate the mechanical properties at micrometer scale. Besides condensed matter, such technique has also been used to investigate soft materials [9-12]. The way the sample responses to external stresses depends on its elastic and viscous terms. However, the complex structure and composition in soft matter systems make it challenging to investigate accurately the distribution of applied stresses through their interior. Many soft matter systems hold large colloidal aggregations or long polymeric chains, which have an essential mechanical role [4]. In living cells, for example, the cytoskeleton and the cytoplasmic fluid are constantly exchanging momentum with the extracellular surroundings $[13,14]$. The knowledge of why these organelles influence the cell stiffness differently in healthy and sick cells, may lead to treatments for several diseases [15].

To investigate the link between mesoscopic and macroscopic features, we employ a molecular coarse-grained approach to reproduce rheological behavior of soft matter. We design a viscoelastic material composed of a particle-spring network immersed in a viscous medium where the contribution of elastic and viscous interactions can be controlled at the mesoscopic level. This is compatible with suspended polymer chains, colloidal aggregations, and other load-bearing structures as commonly found in soft materials [4]. Although real materials unlikely present purely quadratic potentials, for small deviations, molecular interactions displaying a potential well can be described appropriately by a spring-like interaction. For instance, the equivalent spring constant of the Lennard-Jones potential can be calculated as $72 \epsilon_{0} / \ell_{0}^{2}$, where 


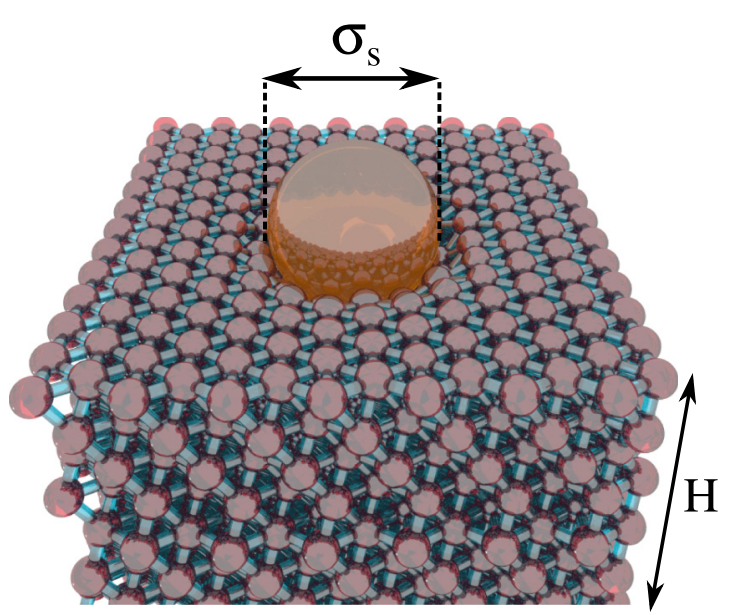

FIG. 1. Particle-spring network arranged in an FCC (facecentered cubic) lattice with height $H$ and immersed in a viscous medium (not shown in the figure). Particles are bonded to their 12 nearest neighbors. A spherical indenter of diameter $\sigma_{s}$ is used to press down the top surface of the network.

$\epsilon_{0}$ is the height of the potential well and $\ell_{0}$ the equilibrium distance between molecules [16]. Moreover, our model is easily changed to support a more realistic potential in order to study more complex materials. We also consider the spring network as a regular lattice. Although complex structures may influence rheological properties of a material, simple geometries have been successfully applied to a large number of problems in mesoscopic models of condensed $[17,18]$ and soft materials [19-21].

\section{NUMERICAL MODEL}

Our computational model consists of $N$ spherical particles of diameter $\sigma$ and mass $m$ arranged in a face-centered cubic (FCC) lattice with a height given by $H$ and a bottom and top plane given by $20 \sigma \times 20 \sigma \sin (\pi / 3)$. Every particle interacts with its 12 nearest neighbors by a spring potential. This elastic network is immersed in a medium of viscosity $\mu$. Viscous effects are taken into account by considering friction between the particles and the medium. The network is indented by a hard sphere of diameter $\sigma_{s}$, as shown in Fig. 1. This spherical indenter moves down with constant speed until a maximum indentation depth $\delta_{0}$ is achieved. To avoid nonlinearities, we consider $\delta_{0}=\sigma$, i.e., the maximum indentation is equal to the thickness of one layer of particles. Once in contact, the indenter applies a stress onto the particles. The bottom layer of the network is in contact to a hard substrate, where particles cannot move down but are free to slide horizontally. The viscous flow when the fluid is squeezed is not considered. However, it has been shown that such effects in deforming porous media become relevant only in the very beginning of the force ramp, at small timescales [14,22]. After that, rheological macroscopic response is dominated by viscoelastic properties.

The equation of motion of the $i_{t h}$ particle is given by the following Langevin-like equation [23]:

$$
m \frac{d^{2} \vec{r}_{i}}{d t^{2}}=-\gamma \vec{v}_{i}-\nabla U_{i}
$$

where $\vec{r}_{i}$ and $\vec{v}_{i}$ are the position and velocity vectors of particle $i$, respectively. The term $\gamma \vec{v}_{i}$, known as Stoke's law, describes the drag force acting on spherical bodies, where the coefficient of friction is given by $\gamma=3 \pi \sigma \mu$ [24]. Although similar, it is not exactly the Langevin equation since the random forces applied to the particles are not due to the molecules of the fluid, but, instead, to other particles in the medium. The potential $U_{i}$ of particle $i$ due to the neighboring particles and the indenter is given by

$$
U_{i}=\sum_{j} \frac{k_{i j}\left(r_{i j}-\ell\right)^{2}}{2}+\epsilon\left(\frac{\sigma}{r_{i s}-\left(\sigma_{s}-\sigma\right) / 2}\right)^{\alpha},
$$

where $r_{i j}=\left|\vec{r}_{i}-\vec{r}_{j}\right|$ is the distance and $\ell$ the equilibrium distance between particles $i$ and $j$, bonded by the spring constant $k_{i j}$. The last term of Eq. (2) applies only to surface particles in contact with the indenter, where $r_{i s}=\left|\vec{r}_{i}-\vec{r}_{s}\right|$ is the distance between the particle $i$ and the indenter, $\epsilon$ is a constant of energy, and $\alpha$ regulates the hardness of the indenter. The particles and the indenter exclusively interact through this hard-sphere potential with a high value of $\alpha$. See the constants used in Appendix A.

The equations of motion shown in Eq. (1) are solved through molecular dynamics simulations with a time integration done by the velocity Verlet algorithm with a time step of 0.001 , and periodic boundary conditions applied to the horizontal plane $[25,26]$. The contact force $F$ is the sum of all collisions on the indenter, computed at each time as the indenter slowly presses down the network. $F$ increases with the indentation depth $\delta$ since more collisions occur on the indenter. The indentation is divided in 120 microindentation steps, until $\delta_{0}$ is reached. These collisions cause a fluctuation in $F$, but an equilibrium state is reached in around $10^{5}$ time steps. After equilibrium, we perform an additional $2 \times 10^{5}$ time steps to average the quantities of interest.

\section{INDENTATION ASSAYS IN ELASTIC NETWORKS}

Before viscoelastic materials, we explore networks without local friction $(\gamma=0)$. Initially, we study homogenous networks, where all bonds have the same spring constant, $k_{i j}=k$, and later we investigate the role of heterogeneities of local elasticity in the global behavior.

\section{A. Homogeneous networks}

The distribution of stress into an elastic sample, due to an indentation $\delta$, can be described by the Cauchy stress tensor [27], whereas the force experienced by a spherical indenter is well described by the Hertz model

$$
F_{H}=\frac{8 \sqrt{2}}{9} E \sqrt{\sigma_{s}} \delta^{3 / 2}
$$

where $E$ is the effective elastic modulus of the material [28]. The contact forces computed in elastic networks perfectly agree with the Hertz model, where $E$ can be obtained by fitting the numerical data with Eq. (3), keeping the other parameters fixed. The results for homogeneous elastic networks are shown in Fig. 2. As expected for this simple network, the effective modulus of elasticity scales linearly with $k$. In addition, $E$ is proportional to the sample height in the 

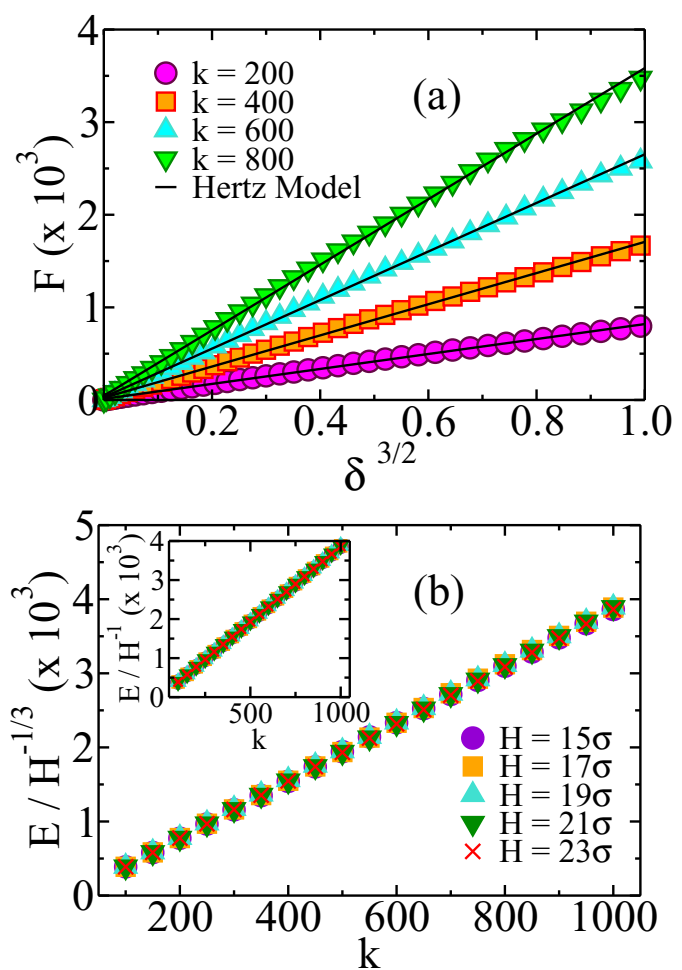

FIG. 2. (a) The contact force, $F$, probed by a spherical indenter as a function of the indentation depth, $\delta$, in homogeneous elastic networks for different value of $k$ and $H=15 \sigma$. The elastic modulus $E$ is computed by fitting our numerical data with the Hertz model (straight lines), shown in Eq. (3), in different elastic networks. (b) As expected for homogeneous materials, $E$ displays a linear behavior with $k$. Moreover, $E$ is proportional to the sample height in the form $E \propto H^{-\zeta}$, where $\zeta$ depends on the indenter geometry. Spherical and flat indenters, for instance, give $\zeta=1 / 3$ (main graph) and 1 (inset), respectively.

form $E \propto H^{-\zeta}$, where the exponent $\zeta=1 / 3$, for spherical indenters, and $\zeta=1$, for flat indenters. In the latter case, the deformation is uniaxial where $E$ represents the Young's modulus of the material and $\zeta$ can be found analytically with springs in series. However, for spherical indenters, $E$ seems to take into account also the shear modulus of the material.

\section{B. Comparison to virial stress}

In the general form, the second-order stress tensor $\overline{\bar{\sigma}}$ in a continuum medium can be written as

$$
\overline{\bar{\sigma}}=\left(\begin{array}{lll}
\sigma_{x x} & \sigma_{x y} & \sigma_{x z} \\
\sigma_{y x} & \sigma_{y y} & \sigma_{y z} \\
\sigma_{z x} & \sigma_{z y} & \sigma_{z z}
\end{array}\right),
$$

where element $\sigma_{p q}$ measures the rate at which $q$-directed momentum is transported in the direction $p$.

The virial stress is an atomistic definition for stress that is equivalent to the continuum Cauchy stress in which each element of the tensor is composed of a kinetic part and a
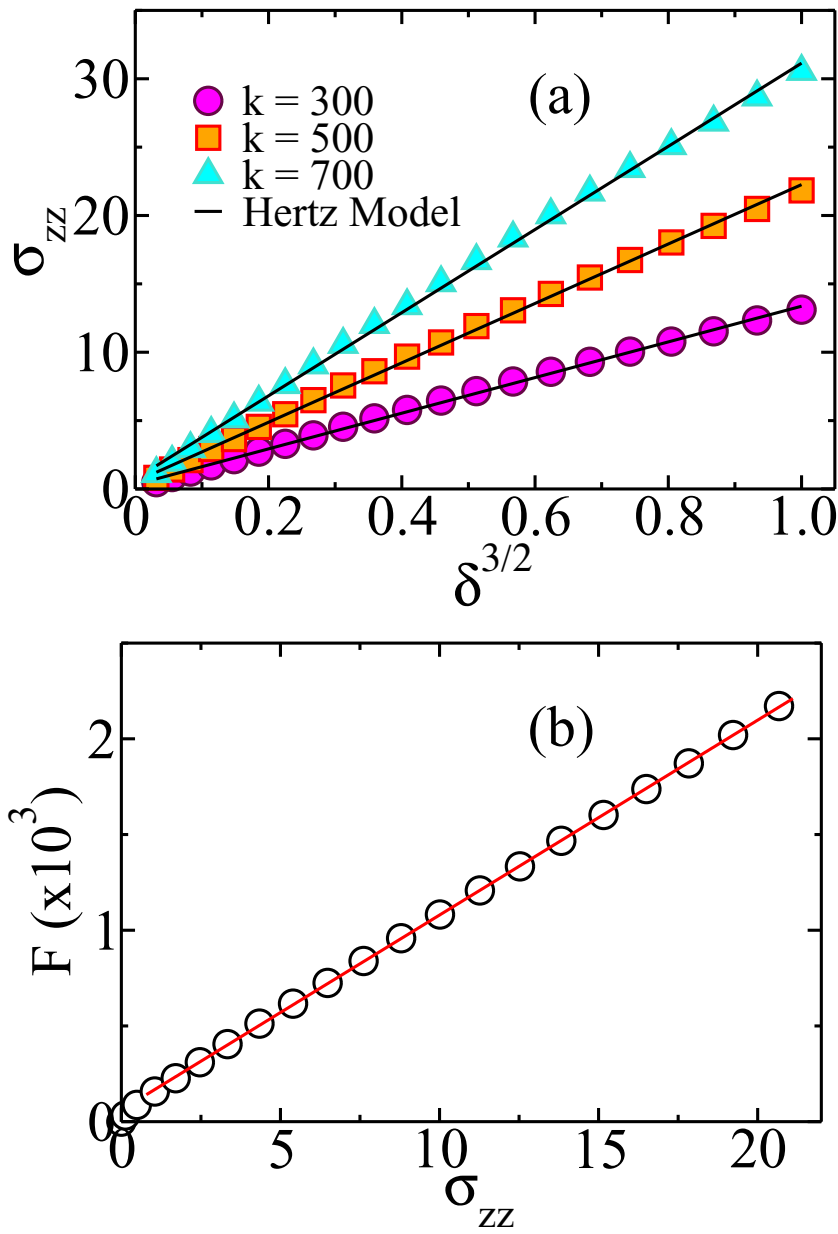

FIG. 3. (a) The $z z$ element of the virial stress tensor $\sigma_{z z}$ vs the indentation to the power of $3 / 2, \delta^{3 / 2}$, for different values of $k$ and $\gamma=0$. The straight lines represent the Hertz behavior. (b) The contact force $F$ vs $\sigma_{z z}$ at the maximum indentation $\delta=\delta_{0}$ and $k=$ 500. The solid line is a straight line showing linear relation.

potential part averaged over all particles, as follows:

$$
\sigma_{p q}=\frac{1}{V}\left(m \sum_{i}^{N} v_{i p} v_{i q}+\frac{1}{2} \sum_{i \neq j}^{N} r_{i j q} \tau_{i j q}\right),
$$

where $m$ is the mass and $V$ the volume occupied by each particle, $v_{i p}$ is the component $p$ of the velocity of particle $i$, $r_{i j q}$ is the component $q$ of the vector $\vec{r}_{i j}$ separating particles $i$ and $j, \tau_{i j q}$ is the component $q$ of the force exerted on particle $i$ by particle $j[29]$. For instance, the $z z$ element is written as

$$
\sigma_{z z}=\frac{1}{V}\left(m \sum_{i}^{N} v_{i z}^{2}+\frac{1}{2} \sum_{i \neq j}^{N}\left(z_{i}-z_{j}\right) \tau_{i j z}\right),
$$

where $z_{i}$ is the position $z$ of particle $i$.

In order to show that the virial stress in our system is equivalent to the Hertz model we compare the component of the virial stress tensor $\sigma_{z z}$ to the total force $F$ on the indenter, since $F$ obeys the Hertzian deformation theory and that, in our computational experiment, the indenter is pushing the soft sample in $z$ direction. Figure 3(a) shows that $\sigma_{z z}$ holds the 
same power-law behavior than that of the Hertz model as the indentation increases. We also show that $\sigma_{z z}$ is proportional to $F$ at the maximum indentation, see Fig. 3(b). These two behaviors are strong evidences that our microscopic model reproduces well the elastic response predicted by the continuum solid deformation theory.

\section{Heterogeneous networks}

For simplicity, we consider heterogeneous networks made of only two types of bonds with spring constants given by $k_{1}$ and $k_{2}$, respectively. Moreover, this binary network is built in two fashions depending on the distribution of $k_{1}$ and $k_{2}$, namely, the double-layered and the binary random network. In the first, two homogenous layers, with spring constants given by $k_{1}$ and $k_{2}$, respectively, are deposited one on the top of the other. In the second, $k_{i j}$ is randomly assigned to $k_{1}$ or $k_{2}$ according to probabilities $\phi_{1}$ and $\phi_{2}$, respectively. In both cases, $\phi_{1}$ and $\phi_{2}$ also stand to the fraction of each bond and $\phi_{1}+\phi_{2}=1$. For the limit cases of $\phi_{1}=0$ and $\phi_{1}=1$, both the double-layered and the binary random networks become homogeneous with spring constant $k_{2}$ and $k_{1}$, respectively.

The effective elastic modulus in heterogenous network as a function of $\phi_{1}$ is shown in Fig. 4. In double-layered networks, $E$ grows with a cubic function given by $E=a+$ $b \phi+c \phi^{2}+d \phi^{3}$. On the other hand, when $k_{1}$ and $k_{2}$ are randomly distributed over the network, $E$ follows a straight line, $E=a+e \phi$. The constants $a, b, c, d$, and $e$ depend on $k_{1}$ and $k_{2}$ as well as on geometrical parameters. Interestingly, the random distribution of local stiffness vanishes those polynomial higher order found in double-layered networks.

\section{RELAXATION ASSAYS IN VISCOELASTIC NETWORKS}

In order to study the effects of viscous forces $(\gamma>0)$ in macroscopic rheological behaviors, we perform relaxation and oscillatory assays in homogenous viscoelastic networks. In relaxation experiments, the indenter is initially located above the network and moving down into the top surface. Before touching the sample, the contact force is zero. After contact, at time $t_{1}=0$, the force rises until the maximum indentation depth, $\delta_{0}$, is achieved, at $t_{3}$. Figure 5(a) shows the time evolution of the normalized contact force, $f$, for $k=$ 500 and several values of $\gamma$. The loading time, $\tau_{l}=t_{3}-t_{1}$, is the time the indenter pushes the sample. After reaching $\delta_{0}$, the indenter stops moving, but the sample particles may continue to move subjected to friction (the dwell stage). When viscous forces are negligible (elastic networks), the particles immediately rearrange themselves to an equilibrium state and thus $f$ becomes nearly constant, except for some noise due to the perpetual undamped movement of the particles. On the other hand, in viscoelastic networks, this noise quickly vanishes while $f$ decreases continuously to the value of the undamped case, at $t_{4}$, since particles need time to relax into the equilibrium state. For long times, networks with the same elastic properties experience the same contact force, regardless of the viscous contribution.

Our numerical results are compatible with the Standard Linear Solid (SLS) model composed of a Maxwell material
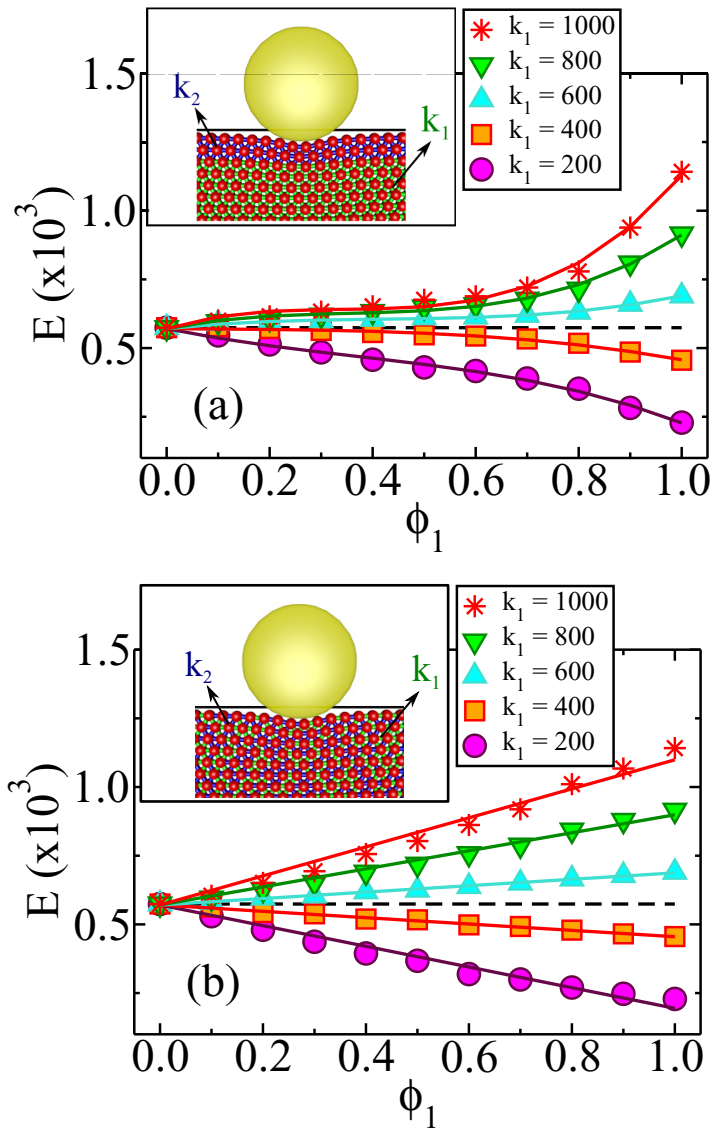

FIG. 4. The effective modulus of elasticity, $E$, as a function of the fraction of $k_{1}$-bonds in heterogeneous elastic networks, $\phi_{1}$, for $k_{2}=500$ and several values of $k_{1}$. The double-layered network is shown in (a) while the binary random network in (b). The snapshots in the insets shows how $k_{1}$ and $k_{2}$ are distributed in the each network. The black dashed lines in each graph represent the elastic modulus of homogeneous networks, for $k_{1}=k_{2}=500$, with $E=570.75$. $E$ decreases with $\phi_{1}$ for $k_{1}<k_{2}$, but increases with $\phi_{1}$ for $k_{1}>k_{2}$, regardless the distribution of $k_{1}$ and $k_{2}$. Cubic functions and straight lines, shown in colored solid lines, are used to fit each set of points in (a) and (b), respectively. In double-layered networks, $E$ grows with a cubic function given by $E=a+b \phi+c \phi^{2}+d \phi^{3}$. On the other hand, when $k_{1}$ and $k_{2}$ are randomly distributed over the network, $E$ changes according to the straight line $E=a+e \phi$. The constants $a=570.75, b, c, d$, and $e$ depend on $k_{1}$ and $k_{2}$ as well as on geometrical parameters.

(i.e., a spring of elastic modulus $E_{0}$ in series with a dashpot of viscosity $\eta$ ) in parallel to another spring of elastic modulus $E_{\infty}$, see Fig. 5(b). Such analytical model successfully describes rheological behaviors of a large number of soft materials, such as polymers [30], soft gels [31], and living cells [32,33]. The relaxation function of this model is know as $R(t)=E_{\infty}+E_{0} e^{-t / \tau}$, where the relaxation time is given by $\tau=\eta / E_{0}$. Initially, the effective modulus of elasticity is given by the sum of $E_{\infty}$ and $E_{0}$, but, for long times, the influence of $E_{0}$ vanishes, leaving $E_{\infty}$ to dominate the elasticity of the material, regardless of the relaxation time. Notice that $E_{\infty}$ corresponds to the elastic modulus computed previously in elastic networks. The analytical force curves in the SLS 


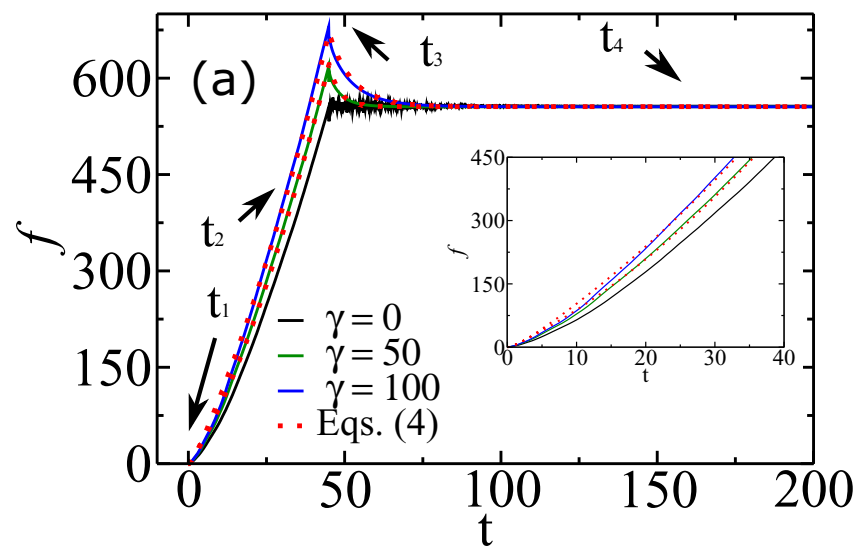

(b)

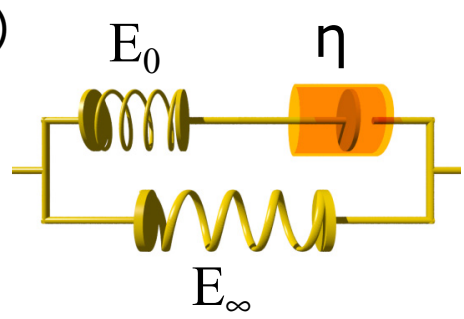

FIG. 5. Relaxation experiments in viscoelastic networks, for $k=$ 500 and different values of $\gamma$, are shown in (a). The contact force increases in the loading stage and decreases in the dwell stage. The force is normalized by geometric constants; see Appendix B. Our numerical results are compatible with the standard linear solid (SLS) model given in Eqs. (4). The inset shows the numerical and the analytical results diverging a little for small times before converging again (for $t \gtrsim 20$ ). A schematic illustration of the SLS model is shown in (b), with a dashpot of viscosity $\eta$ and two springs of elastic moduli $E_{0}$ and $E_{\infty}$.

model with a spherical indenter can be obtained separately in the loading and dwell stages, respectively, as follows (see Appendix B)

$$
\begin{aligned}
& f_{l}(t)=a_{1} t^{3 / 2}+a_{2} \sqrt{t}+a_{3} \operatorname{erf}\left(\sqrt{\frac{t}{\tau}}\right) e^{-t / \tau}, \quad t \leqslant \tau_{l}, \\
& f_{d}(t)=a_{4}+a_{5} e^{-\left(t-\tau_{l}\right) / \tau}+a_{6} e^{-t / \tau}, \quad t \geqslant \tau_{l},
\end{aligned}
$$

where $\operatorname{erf}(t)$ is the error function and the constants are given by

$$
\begin{aligned}
& a_{1}=\frac{E_{\infty}}{\tau_{l}^{3 / 2}}, \quad a_{2}=\frac{3}{2} \frac{\tau}{\tau_{l}^{3 / 2}} E_{0}, \quad a_{3}=-\frac{3}{4} \sqrt{\pi} E_{0}\left(\frac{\tau}{\tau_{l}}\right)^{3 / 2}, \\
& a_{4}=E_{\infty}, \quad a_{5}=\frac{3}{2} \frac{\tau}{\tau_{l}} E_{0}, \quad a_{6}=a_{3} \operatorname{erf}\left(\sqrt{\frac{\tau_{l}}{\tau}}\right) .
\end{aligned}
$$

Combining our numerical results with Eqs. (4) allows us to find the relations between macroscopic ( $\tau$ and $E_{0}$ ) and microscopic parameters $(k$ and $\gamma$ ) in viscoelastic networks. As shown for elastic networks, $E_{\infty}$ increases linearly with $k$ and does not depend on $\gamma$. We find that $\tau$ decreases with $k$ and increases with $\gamma$ [see Fig. 6(a)]. On the other hand, $E_{0}$ increases with $k$ but decreases with the local dissipation [see Fig. 6(b)]. Interestingly, high values of $k$ leads to the same value of $\tau$, regardless of $\gamma$, as high values of $\gamma$ leads to the
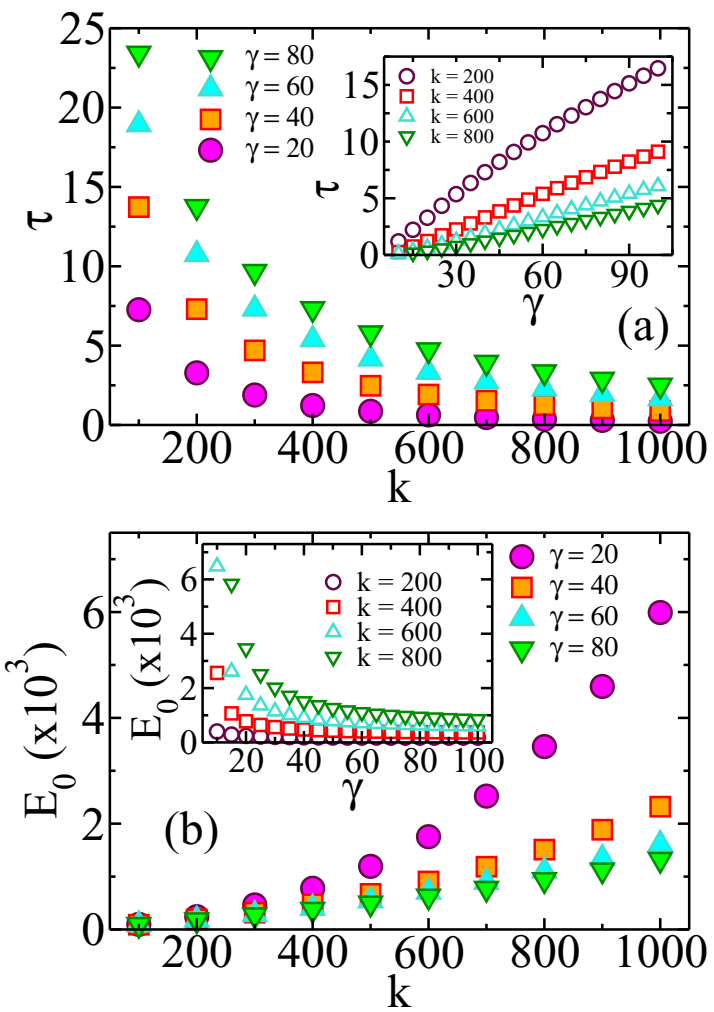

FIG. 6. The macroscopic properties $\tau$ and $E_{0}$, in (a) and (b), respectively, as a function of microscopic parameters, $k$ and $\gamma$, in viscoelastic networks under relaxation assays.

same value of $E_{0}$, regardless of $k$. Due to the linear deformation regime and the harmonic interactions, the presence of a thermal bath would only rise fluctuations (see Appendix C). Nonlinear effects, however, should lead to intriguing results.

\section{Comparison to atomic force microscopic measurements}

In order to validate our numerical results, we performed atomic force microscopic (AFM) experiments in polyacrylamide gels immersed in water, where different mechanical properties can be achieved by tuning the concentration of bisacrylamide. In particular, it was recently demonstrated that polyacrylamide gels are well described by the standard linear solid model $[34,35]$.

Polyacrylamide gels were prepared from the stock solution of $30 \%$ acrylamide (by weight) in two different concentrations of N,N-bis acrylamide, namely, $0.2 \%$ and $0.8 \%$ in volume. The final gels have a concentration of $0.375 \mathrm{M}$ Tris-HCL, with $\mathrm{pH}$ 8.8. The gels were polymerized chemically by the addition of $10 \mu \mathrm{L}$ of tetramethylethylenediamine (Temed) and $0.1 \mathrm{~mL}$ of $15 \%$ ammonium persulfate solution $/ 10 \mathrm{~mL}$ of gel solution. Conventional force curves were then measured in an AFM (MFP-3D, Asylum-Research, Santa Barbara, CA, USA) coupled to an inverted optical microscope Nikon IX51. Soft cantilevers with nominal spring constant of $0.02 \mathrm{~N} / \mathrm{m}$ were used to probe the gels. AFM tips have pyramidal shapes with half-opening angles of $38^{\circ}$. To reduce adhesion effects in the cantilever, the force measurements were performed in distilled water at room temperature such that the gel and the AFM tip were fully immersed in water. We obtained gels with average 

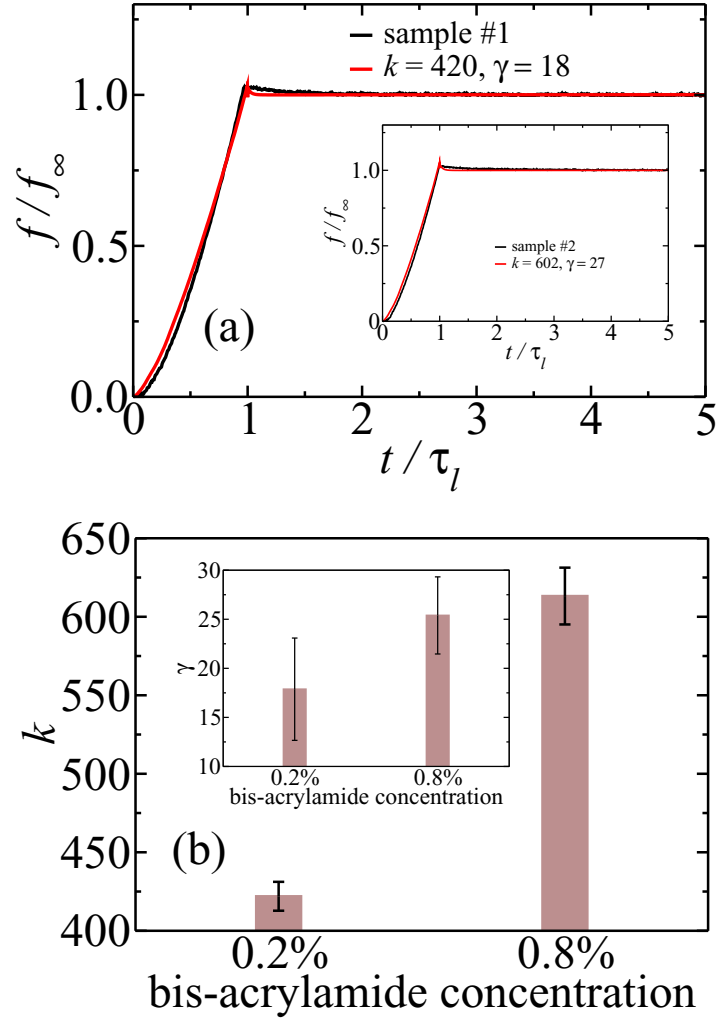

FIG. 7. Experimental relaxation curves of two samples of polyacrylamide gels with different concentration of bisacrylamide. The experimental curves (black lines) are compared with relaxation curves obtained numerically (red lines) in our model with specific values of $k$ and $\gamma$. The force $f$ is normalized by $f_{\infty}$, the value of $f$ for long times, whereas the time $t$ is normalized by the loading time $\tau_{l}$. Sample 1 (in the main graph) is best fitted for $k=420$ and $\gamma=18$, while sample 2 (in the inset) for $k=642$ and $\gamma=27$. The average of $k$ and $\gamma$ over several samples are shown in (b).

stiffness of approximately $8 \mathrm{kPa}$ and $39 \mathrm{kPa}$ for the gels with $0.2 \%$ and $0.8 \%$ of bisacrylamide, respectively.

Figure 7(a) shows the comparison between experimental and numerical force curves for two samples with different concentration of bisacrylamide: "sample 1 " for $0.2 \%$, and "sample 2" for $0.8 \%$. The experimental relaxation curves present a good agreement with those obtained numerically, where the equivalent $k$ and $\gamma$ can be found for each sample. Figure 7(b) shows $k$ and $\gamma$ averaged over different samples for each concentration considered. The concentration of bisacrylamide clearly increases the average of both $k$ and $\gamma$.

\section{OSCILLATORY ASSAYS IN VISCOELASTIC NETWORKS}

In oscillatory experiments, instead of stoping the indenter after $\delta_{0}$ is achieved, a sinusoidal strain is imposed with the indentation depth following the function, $\delta(t)=\delta_{0}+\delta_{a} \sin (\omega t)$, where $\delta_{a}\left(\delta_{a}=\sigma\right)$ is the amplitude and $\omega$ the angular frequency of oscillation. The response force also presents a periodic behavior but with a time delay that depends on viscoelastic properties, $F=F_{0}+F_{a} \sin (\omega t+\lambda)$, where $F_{0}$ is the force at $\delta_{0}, F_{a}$ is the amplitude of the force, and $\lambda$ is the
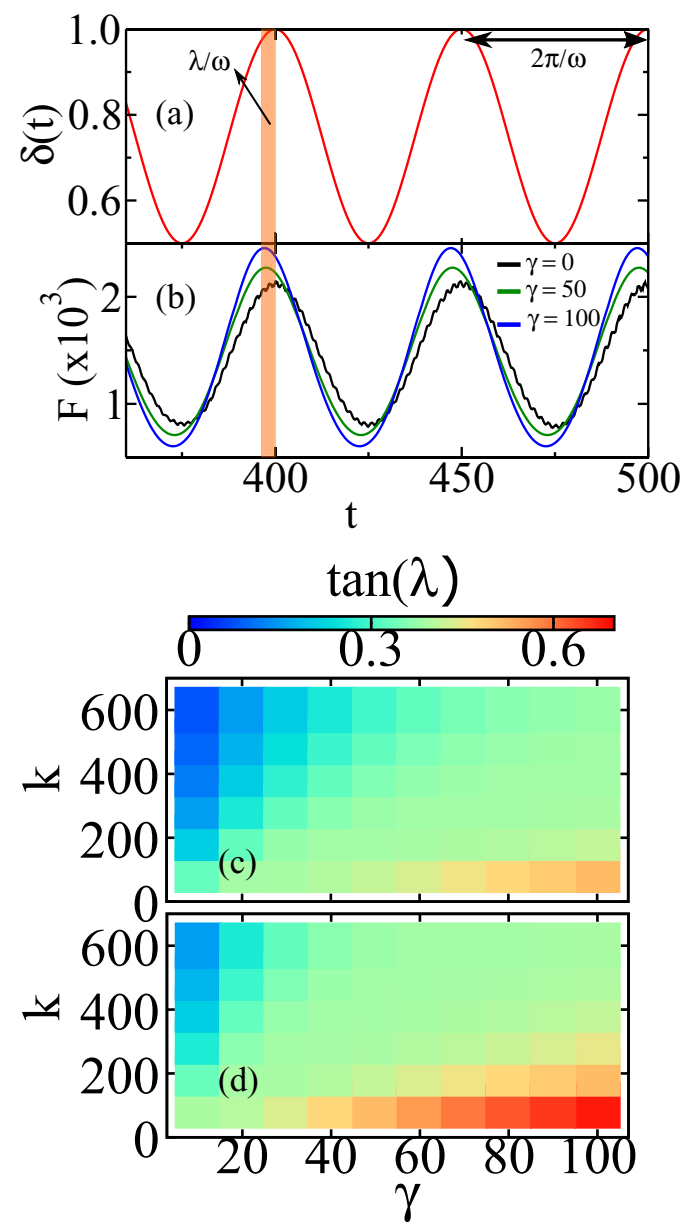

FIG. 8. Oscillatory experiments in homogeneous viscoelastic networks. A sinusoidal indentation, $\delta$, shown in (a) is applied to the network for $k=500$ and different values of $\gamma$. Corresponding response forces, $F$, are shown in (b). The orange dashed lines highlight the phase lag, $\lambda$, between $F$ and $\delta$, which depends on viscoelastic properties. We also show color maps of $\tan (\lambda)$ for different values of $k$ and $\gamma$ for $\omega=0.02$ (c) and for $\omega=0.04$ (d).

phase lag between force and indentation. Some of these curves are shown in Figs. 8(a) and 8(b). The phase lag vanishes for $\gamma=0$, as it is expected for elastic materials, but increases with $\gamma$, for viscoelastic materials.

The tangent of $\lambda$ defines the ratio between the loss modulus, $G^{\prime}=\frac{\delta_{a}}{F_{a}} \sin (\lambda)$, and the storage modulus, $G^{\prime \prime}=\frac{\delta_{a}}{F_{a}} \cos (\lambda)$, and also represents a quantitative way to define the fluidity of viscoelastic materials, i.e., $\tan (\lambda)<1$ leads to solidlike materials while $\tan (\lambda)>1$ to fluid-like materials. High values of $\omega$ favor the fluidity of the material, as shown in the panel of Fig. 9 for several values of $\gamma$. Figures 8(c) and 8(d) shows color maps of $\tan (\lambda)$ for more values of $k$ and $\gamma$. We only observe viscoelastic solids in all cases of $k$ and $\gamma$ considered in this work. Fluidlike behavior should be observed for very high values of $\omega$, which requires a time step too small compared to the time of the experiment. Moreover, viscoelastic materials may present different relaxation times regarding of how the sample is deformed.

In this work, we obtained $\tau$ and $\tau_{o}$ in relaxation and oscillatory conditions, respectively. Standard linear solids under 


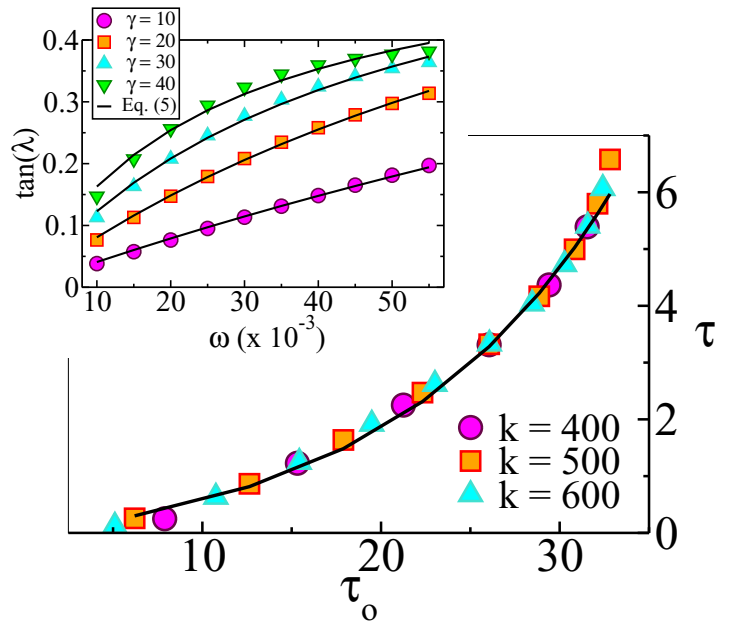

FIG. 9. In oscillatory experiments, the tangent of the phase lag $\lambda$ as a function of $\omega$ is shown in the panel for $k=500$ and different values of $\gamma$. Our results recover in a good agreement the analytical behavior of $\tan (\lambda)$ given in Eq. (5), where the relaxation time in oscillatory experiments, $\tau_{o}$, is obtained. The relaxation times, $\tau$ and $\tau_{o}$, obtained in different experiments of deformation, depend on each other as follows, $\tau=0.466\left(e^{0.08 \tau_{o}}-1\right)$ (shown in black solid line), regardless of $k$ and $\gamma$, as shown in the main graph.

oscillatory strain leads to the following behavior [8]

$$
\tan (\lambda)=A \frac{\omega \tau_{o}}{1+\omega^{2} \tau_{o}^{2}},
$$

which is used to fit those numerical data in Fig. 9 in order to obtain $\tau_{o}$, where $A$ is a material constant. The main graph in Fig. 9 shows that $\tau$ and $\tau_{o}$ depend on each other by the following exponential relation, $\tau=0.466\left(e^{0.08 \tau_{o}}-1\right)$. Each point in the graph represents a different material, with different $k$ and $\gamma$, but they all collapse on that relation, suggesting some kind of universal behavior.

The behavior of Eq. (5) shows that the loss tangent increases from zero to a maximum value and then gradually decreases to zero. The reason is that as the frequency increases the relative motion between the fluid and the particles also increases which in turn yields increased dissipation. However, beyond the peak, the drag forces on the particles become so great that the fluid and the network move together and the dissipation decreases [36]. In our model, the lack of the fluid redistribution through the network may lead to an undervaluation of viscous effects at high frequencies. However, the very satisfactory agreement (shown in the panel of Fig. 9) between our numerical data and the analytical behavior of viscoelastic solids, given by Eq. (5), suggests this misestimation is not relevant for the set of parameters used in this work. In fact, the frequencies used here are much lesser than the peak value, and the loss tangent is far away to reach the maximum.

\section{CONCLUSION}

In conclusion, we performed different indentation experiments to study macroscopic rheological behaviors in viscoelastic soft materials in terms of their microscopic elastic and viscous constituents, namely, the local stiffness and the coefficient of friction. We showed that homogeneous elastic networks recover the Hertz theory of mechanical contact and that the effective modulus of elasticity, $E$, changes with the network height, $H$, as follows $E \propto H^{-\zeta}$, where the exponent depends on the indenter geometry. Heterogeneous elastic networks were built with only two spring constants, $k_{1}$ or $k_{2}$. When $k_{1}$ and $k_{2}$ are separated in layers, $E$ follows a cubic function with the fraction of $k_{1}$ bonds, $\phi_{1}$, however, when $k_{1}$ and $k_{2}$ are randomly distributed over the network, the higher order terms vanish and $E$ changes linearly with $\phi_{1}$. Viscoelastic networks are compatible with the analytical standard linear solid (SLS) model, described by two elastic moduli, $E_{0}$ and $E_{\infty}$, and a relaxation time, $\tau$. Therefore, our work applies to all those materials described by the Hertz and SLS models, such as polymers, gels and living cells. Besides, our results were validated both by experiments and by analytical description of continuum media. In oscillatory experiments, the phase lag, $\lambda$, between force and indentation and the relaxation time, $\tau_{o}$, are computed. We showed how all these macroscopic properties $\left(E_{0}, E_{\infty}, \tau, \lambda\right.$, and $\left.\tau_{o}\right)$ depend on the microscopic parameters. In addition, the interplay between $\tau$ and $\tau_{0}$, obtained in different experiments, follows an exponential relation, regardless of the mesoscopic interactions. All viscoelastic networks studied here collapsed on this curve, suggesting a universal behavior in viscoelastic materials. A future work shall unveil why it presents an exponential form. However, this brings to light an internal mechanism of how soft matter works in different scales. We believe our computational model is a robust framework being able to lay the groundwork to investigate even more complex and nonlinear soft materials.

\section{ACKNOWLEDGMENTS}

We acknowledge financial support from the Brazilian agencies CNPq, CAPES, FUNCAP. This work was also supported by the Serrapilheira Institute (grant No. Serra-1709-18453).

\section{APPENDIX A: PARAMETERS USED IN THE NUMERICAL MODEL}

It is usual in molecular dynamics (MD) simulations to describe quantities in dimensionless form. The following are some values used in this work. Network parameters: $\sigma=$ $1 ; m=1 ; \ell=1 ; H=15 \sigma$, except in Fig. 2(b), where $H$ is changed; and $N=7580$, except in Fig. 10 where $N$ is changed. The indenter radius is $\sigma_{s}=11 \sigma$ (see Fig. 10). In the particle-indenter interaction: $\epsilon=1$ and $\alpha=800$. The reason for the high value of $\alpha$ is to mimic the discontinuous behavior of hard-core potentials, typically found in very narrow-ranged interactions. Such numerical artifact has been used in several works [37-39].

In order to simulate real materials, one may scale MD parameters based on actual forces curves. Doing this, we can adopt the following strategy: AFM force curves are fitted with Eqs. (4) to determine the loading time $\tau_{l}$, relaxation time $\tau$, and the rheological parameters $E_{0}$ and $E_{\infty}$. The maximum indentation depth, $\delta_{0}$, is also known from the experiment. In our MD simulations, the maximum indentation depth is equal to $\sigma$ (the diameter of a pseudoatom) and a typical simulation 


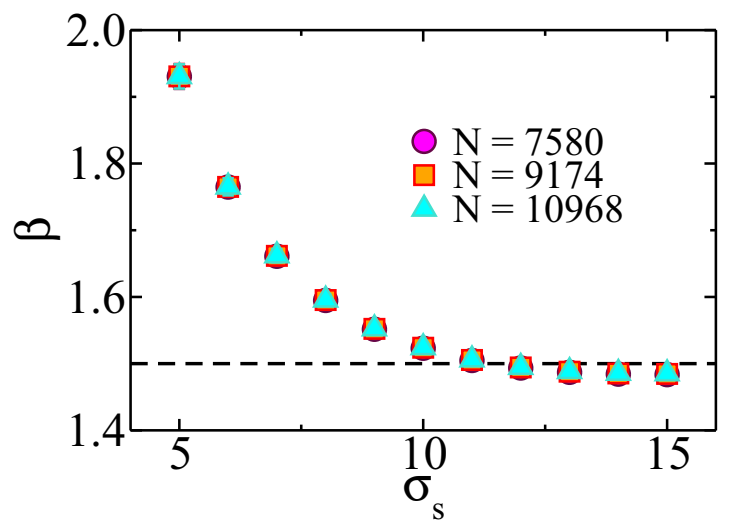

FIG. 10. The contact force $F$ between a spherical indenter and an elastic network, for height $H=15 \sigma$ and different numbers of particles, $N . F$ is a power law of the indentation depth, $F \propto \delta^{\beta}$, where the exponent $\beta$ depends on the indenter geometry [28]. This theory is based on the assumption that the sample is an elastic continuum half-space and the sample deformation is within the linear elastic regime. For conical, spherical and flat indenters, $\beta$ is given by $2,3 / 2$, and 1 , respectively. For large $\sigma_{s}$, the sample experiences a force compatible with a flat indenter whereas the exponent of $\delta$ approaches the unity, while for small $\sigma_{s}$, the sample experiences a force compatible with a conical indenter and the exponent approaches 2 . In our model, we find that $\sigma_{s}=11 \sigma$ is an intermediate value that recovers the exponent $3 / 2$ for spherical indenters.

takes $N_{t}=120 \mathrm{MD}$ time steps. Thus we adopt $\sigma=\delta_{0}$ as our length scale and $t_{0}=\tau_{l} / N_{t}$ as the length of the MD time steps.

In scaled units, the force, elasticity moduli, spring constant, friction coefficient, and the time step take, respectively, the following forms $F_{0}=\frac{\varepsilon}{\sigma}, E=\frac{\varepsilon}{\sigma^{3}}, k=\frac{\varepsilon}{\sigma^{2}}, \gamma=\frac{\varepsilon t_{0}}{\sigma^{2}}$, and $t_{0}=$ $\sigma \sqrt{\frac{m_{0}}{\varepsilon}}$, where $\varepsilon$ is a dimensionless unit of energy used to set interacting potential between pseudo-atoms. To qualitatively reproduce the AFM forces curves measured in polyacrylamide gels with MD simulations, we obtain the following quantities in physical dimensions $\sigma=3.8933 \times 10^{-7} \mathrm{~m}, m_{0} \approx 2.9 \times$ $10^{-12} \mathrm{~kg}, t_{0} \approx 7 \times 10^{-4} \mathrm{~s}, \varepsilon=576.35 \times 10^{-21} \mathrm{~J}, k \approx 4 \times$ $10^{-3} \mathrm{~N} / \mathrm{m}$, and $\gamma \approx 8 \times 10^{-8} \mathrm{~N} \mathrm{~s} \mathrm{~m}^{-1}$.

\section{APPENDIX B: FORCE CURVES DETERMINATION IN THE STANDARD LINEAR SOLID MODEL}

The indentation force curve, at a time $t$, is obtained by solving the following integral:

$$
F(t)=\Omega(\beta) \int_{0}^{t} R\left(t-t^{\prime}\right) \frac{d \delta^{\beta}\left(t^{\prime}\right)}{d t^{\prime}} d t^{\prime},
$$

where $\beta$ and $\Omega(\beta)$ are parameter related to the indenter geometry (see Table I) and $R(t)$ is the time-dependent relaxation function. During load stage, when the sample is pressed down by the indenter with constant velocity, $F$ increases. After reaching the maximum indentation, the indenter stops and the sample relaxes due to its viscoelastic properties leading $F$ to decrease.

For a relaxation experiment, the load (1) and dwell (d) curves are given, respectively, by

$$
\begin{aligned}
f_{l}(t)= & \int_{0}^{t} R\left(t-t^{\prime}\right) \frac{d \bar{\delta}_{l}^{\beta}\left(t^{\prime}\right)}{d t^{\prime}} d t^{\prime}, \quad t \leqslant \tau_{l}, \\
f_{d}(t)= & \int_{0}^{\tau_{l}} R\left(t-t^{\prime}\right) \frac{d \bar{\delta}_{l}^{\beta}\left(t^{\prime}\right)}{d t^{\prime}} d t^{\prime} \\
& +\int_{\tau_{l}}^{t} R\left(t-t^{\prime}\right) \frac{d \bar{\delta}_{d}^{\beta}\left(t^{\prime}\right)}{d t^{\prime}} d t^{\prime}, \quad t \geqslant \tau_{l},
\end{aligned}
$$

where $\tau_{l}$ is the loading time. The indentation history $\delta(t)$ is normalized by the maximum indentation $\delta_{0}$ achieved at $t=\tau_{l}$, such that $\bar{\delta}(t)=\delta(t) / \delta_{0}$, and the force at each stage is also normalized by geometric constants

$$
f(t)=\frac{F(t)}{\Omega(\beta) \delta_{0}^{\beta}} .
$$

In order to solve Eq. (B1), we consider $R(t)$ from the socalled standard linear solid model, given by

$$
R(t)=E_{\infty}+E_{0} e^{-t / \tau},
$$

where $\tau$ is the relaxation time, and $E_{\infty}$ and $E_{0}$ are elastic moduli. The load $\delta_{l}(t)$ indentation profile is well described by a linear function in time, while the dwell profile $\delta_{d}(t)$ is represented by a smooth increasing function in time $g(t)$ (such that $d g / d t \approx 0$ ). More specifically, one can write

$$
\delta_{l}(t)=\delta_{0} \frac{t}{\tau_{l}}, \quad \delta_{d}(t)=\delta_{0}\left[1+g\left(t-\tau_{l}\right)\right],
$$

such that the last term of Eq. (B3) vanishes.

\begin{tabular}{|c|c|c|c|c|}
\hline Geometry & $\beta$ & $\Omega(\beta)$ & contact radius & Obs. \\
\hline flat cylinder & 1.0 & $\frac{2 R}{\left(1-v^{2}\right)}$ & $R$ & $R$ is the indenter radius \\
\hline spherical & 1.5 & $\frac{4}{3} \frac{\sqrt{R}}{\left(1-v^{2}\right)}$ & $\sqrt{R \delta}$ & $R$ is the indenter radius \\
\hline conical & 2.0 & $\frac{2}{\pi} \frac{\tan \Theta}{\left(1-\nu^{2}\right)}$ & $\delta \tan \Theta$ & $\Theta$ is the half-opening angle \\
\hline
\end{tabular}

Replacing (B4) in (B1), we obtain the behavior of force during the load and relaxation processes

$$
\begin{aligned}
& f_{l}\left(t \leqslant \tau_{l}\right)=\int_{0}^{t}\left(E_{\infty}+E_{0} e^{-\left(t-t^{\prime}\right) / \tau}\right) \frac{3}{2 \tau_{l}^{3 / 2}} t^{\prime 1 / 2} d t^{\prime}, \\
& f_{d}\left(t \geqslant \tau_{l}\right)=\int_{0}^{\tau_{l}}\left(E_{\infty}+E_{0} e^{-\left(t-t^{\prime}\right) / \tau}\right) \frac{3}{2 \tau_{l}^{3 / 2}} t^{\prime 1 / 2} d t^{\prime},
\end{aligned}
$$

which lead to Eqs. (4).

TABLE I. Dependence of the parameters $\beta$ and $\Omega(\beta)$ on the indenter geometry. Below, $v$ represents the Poisson ratio and $\delta$ is the indentation. We assume $v=0.5$. 


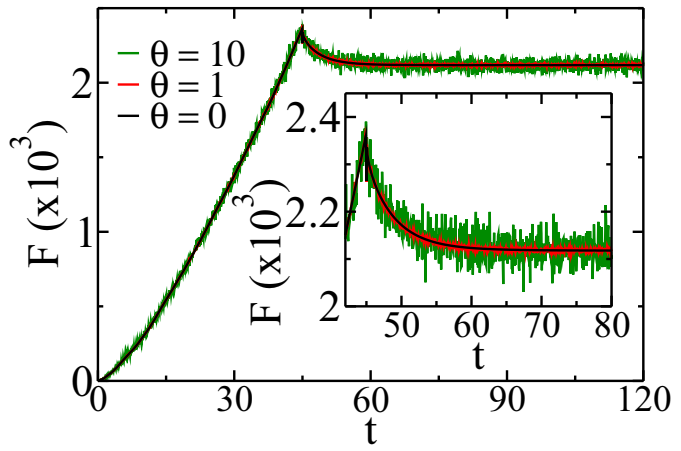

FIG. 11. The force curves for three values of $\theta$ in viscoelastic networks, for $k=500$ and $\gamma=50$. The fluctuations increase with $\theta$, but the mean is clearly the same. The inset graph shows part of these curves, in the beginning of the decay.

\section{APPENDIX C: RELAXATIONS IN VISCOELASTIC NETWORKS: EFFECTS OF A THERMAL BATH}

In our study, we addressed attention only to the linear regime where the indentation is never higher than a single layer of particles. Because of this, and the type of bond interaction (harmonic potential), the effect of temperature is only to rise fluctuations of the particles and the contact force around the values calculated from our original approach (without thermal bath). In fact, the indentation injects energy into the system, which deforms the lattice. This energy is either removed by the friction (when viscous forces are relevant, $\gamma>0$ ) or kept in the system (for $\gamma=0$ ), increasing thermal agitation.

Here we perform additional simulations to study the influence in our results due to the presence of a thermal bath. We run our model with a random noise term in the Langevin approach to represent the presence of a thermal bath, as follows:

$$
m \frac{d^{2} \vec{r}_{i}}{d t^{2}}=-\gamma \vec{v}_{i}-\nabla U_{i}+\vec{\xi}_{i}
$$

where $i$ runs over all particles in the system. These are the same equations of motion shown in Eq. (1). The novelty here is the presence of the noise term $\vec{\xi}_{i}$ which corresponds to an uncorrelated, normally distributed stochastic force with zero mean and finite variance $\theta$.

Figure 11 shows that the inclusion of this thermal noise leads to results similar to the previous ones even when friction takes place (although increasing the agitation of the particles). If the thermal energy is high enough, the particles will vibrate in the lattice's final configuration (final position of the indenter). Therefore, in the linear regime, the omission of the thermal bath in the force curves is not a great issue. A more general approach, however, with nonlinear effects is a relevant problem that should be investigated in a future work.
[1] S. Yip and M. P. Short, Multiscale materials modelling at the mesoscale, Nat. Mater. 12, 774 (2013).

[2] R. J. White, G. C. Y. Peng, and S. S. Demir, Multiscale modeling of biomedical, biological, and behavioral systems (Part 1), IEEE Eng. Med. Biol. Mag. 28, 12 (2009).

[3] P. W. Anderson, More is different, Science 177, 393 (1972).

[4] M. Doi, Soft Matter Physics (Oxford University Press, Oxford, 2013).

[5] M. Praprotnik, L. D. Site, and K. Kremer, Multiscale simulation of soft matter: From scale bridging to adaptive resolution, Annu. Rev. Phys. Chem. 59, 545 (2008).

[6] Z. Qu, A. Garfinkel, J. N. Weiss, and M. Nivala, Multi-scale modeling in biology: How to bridge the gaps between scales? Prog. Biophys. Mol. Biol. 107, 21 (2011).

[7] H. Hertz, Über die Berührung fester elastischer Körper, J. Reine Angew Mathematik 95, 156 (1881).

[8] R. S. Lakes, Viscoelastic Solids (CRC Press, Boca Raton, 2017).

[9] A. F. B. Silva, J. S. Sousa, P. L. R. Cunha, J. V. Lima-Filho, N. M. N. Alencar, C. D. T. Freitas, C. L. N. Oliveira, and M. V. Ramos, Erythrocytes morphology and hemorheology in severe bacterial infection, Mem. Inst. Oswaldo Cruz 114, e190326 (2019).

[10] D. C. Lin and F. Horkay, Nanomechanics of polymer gels and biological tissues: A critical review of analytical approaches in the Hertzian regime and beyond, Soft Matter 4, 669 (2008).

[11] M. Radmacher, Measuring the elastic properties of biological samples with the AFM, IEEE Eng. Med. Biol. Mag. 16, 47 (1997).
[12] L. M. Rebelo, J. S. de Sousa, J. Mendes Filho, and M. Radmacher, Comparison of the viscoelastic properties of cells from different kidney cancer phenotypes measured with atomic force microscopy, Nanotech. 24, 055102 (2013).

[13] M. Gupta, B. R. Sarangi, J. Deschamps, Y. Nematbakhsh, A. Callan-Jones, F. Margadant, R.-M. Mège, C. T. Lim, R. Voituriez, and B. Ladoux, Adaptive rheology and ordering of cell cytoskeleton govern matrix rigidity sensing, Nat. Commun. 6, 7525 (2015).

[14] E. Moeendarbary, L. Valon, M. Fritzsche, A. R. Harris, D. A. Moulding, A. J. Thrasher, E. Stride, L. Mahadevan, and G. T. Charras, The cytoplasm of living cells behaves as a poroelastic material, Nat. Mater. 12, 253 (2013).

[15] J. S. de Sousa, R. S. Freire, F. D. Sousa, M. Radmacher, A. F. B. Silva, M. V. Ramos, A. C. O. Monteiro-Moreira, F. P. Mesquita, M. E. A. Moraes, R. C. Montenegro, and C. L. N. Oliveira, Double power-law viscoelastic relaxation of living cells encodes motility trends, Sci. Rep. 10, 4749 (2020).

[16] D. Kleppner and R. Kolenkow, An Introduction to Mechanics, 2nd ed. (Cambridge University Press, Cambridge, 2014).

[17] C. L. N. Oliveira, A. P. Vieira, H. J. Herrmann, and J. S. Andrade, Jr., Subcritical fatigue in fuse networks, Europhys. Lett. 100, 36006 (2012).

[18] A. A. Moreira, C. L. N. Oliveira, A. Hansen, N. A. M. Araújo, H. J. Herrmann, and J. S. Andrade, Jr., Fracturing Highly Disordered Materials, Phys. Rev. Lett. 109, 255701 (2012).

[19] C. L. N. Oliveira, J. H. T. Bates, and B. Suki, A network model of correlated growth of tissue stiffening in pulmonary fibrosis, New J. Phys. 16, 065022 (2014). 
[20] C. Alves, A. D. Araújo, C. L. N. Oliveira, J. Imsirovic, E. Bartolák-Suki, J. S. Andrade, and B. Suki, Homeostatic maintenance via degradation and repair of elastic fibers under tension, Sci. Rep. 6, 27474 (2016).

[21] C. S. Dias, N. A. M. Araújo, and M. M. Telo da Gama, Nonequilibrium growth of patchy-colloid networks on substrates, Phys. Rev. E 87, 032308 (2012).

[22] Q.-M. Wang, A. C. Mohan, M. L. Oyen, and X.-H. Zhao, Separating viscoelasticity and poroelasticity of gels with different length and time scales, Acta Mech. Sinica 30, 20 (2014).

[23] P. Langevin, Sur la théorie du mouvement brownien, Comptes Rendue Acad. Sci. (Paris) 146, 530 (1908).

[24] G. K. Batchelor, An Introduction to Fluid Dynamics (Cambridge University Press, Cambridge, 1967).

[25] D. C. Rapaport, The Art of Molecular Dynamics Simulation, 2nd ed. (Cambridge University Press, Cambridge, 2004)

[26] J. L. B. de Araújo, F. F. Munarin, G. A. Farias, F. M. Peeters, and W. P. Ferreira, Structure and reentrant percolation in an inverse patchy colloidal system, Phys. Rev. E 95, 062606 (2017).

[27] O. Gonzalez and A. M. Stuart, A First Course in Continuum Mechanics (Cambridge University Press, Cambridge, 2008).

[28] E. K. Dimitriadis, F. Horkay, J. Maresca, B. Kashar, and R. S. Chadwick, Determination of elastic moduli of thin layers of soft material using the atomic force microscope, Biophys. J. 82, 2798 (2002).

[29] J. M. Haile, Molecular Dynamics Simulation - Elementary Methods (Wiley, New York, 1992).

[30] A. Plaseied and A. Fatemi, Deformation response and constitutive modeling of vinyl ester polymer including strain rate and temperature effects, J. Mater. Sci. 43, 1191 (2008).
[31] R. J. Okamoto, E. H. Clayton, and P. V. Bayly, Viscoelastic properties of soft gels: comparison of magnetic resonance elastography and dynamic shear testing in the shear wave regime, Phys. Med. Biol. 56, 6379 (2011).

[32] E. A. G. Peeters, C. W. J. Oomens, C. V. C. Bouten, D. L. Bader, and F. P. T. Baaijens, Viscoelastic properties of single attached cells under compression, J. Biomech. Eng. 127, 237 (2005).

[33] E. J. Koay, A. C. Shieh, and K. A. Athanasiou, Creep indentation of single cells, J. Biomech. Eng. 125, 334 (2003).

[34] Y. Abidine, V. M. Laurent, R. Michel, A. Duperray, L. I. Palade, and C. Verdier, Physical properties of polyacrylamide gels probed by AFM and rheology, Europhys. Lett. 109, 38003 (2015).

[35] J. S. de Sousa, J. A. C. Santos, E. B. Barros, L. M. R. Alencar, W. T. Cruz, M. V. Ramos, and J. Mendes Filho, Analytical model of atomic-force-microscopy force curves in viscoelastic materials exhibiting power law relaxation, J. Appl. Phys. 121, 034901 (2017).

[36] A. M. Wijesinghe and H. B. Kingsbury, On the Dynamic Behavior of Poroelastic Materials, J. Acoust. Soc. Am. 65, 90 (1979).

[37] S. Mossa, F. Sciortino, P. Tartaglia, and E. Zaccarelli, GroundState clusters for short-range attractive and long-range repulsive potentials, Langmuir 20, 10756 (2004).

[38] E. Zaccarelli, Colloidal gels: equilibrium and non-equilibrium routes, J. Phys.: Condens. Matter 19, 323101 (2007).

[39] J. C. F. Toledano, F. Sciortino, and E. Zaccarelli, Colloidal systems with competing interactions: from an arrested repulsive cluster phase to a gel, Soft Matter 5, 2390 (2009). 